\title{
A Wearable Urinalysis Device Based on Inclusive Design Theory
}

$$
\text { Hao-Tian ZHANG }{ }^{1, a} \text {, Zhao-Chu YANG }{ }^{1, b} \text {, and Tao DONG }{ }^{2, c, *}
$$

${ }^{1}$ Institute of Applied Micro-Nano Science and Technology - IAMNST, Chongqing Key Laboratory of Colleges Micro-Nano Systems Technology and Smart Transducing, Chongqing Key Laboratory of Micro-Nano Systems and Smart Transduction, Chongqing Engineering Laboratory for Detection, Control and Integrated System, National Research Base of Intelligent Manufacturing Service, Chongqing Technology and Business University, Chongqing, China

${ }^{2}$ Department of Microsystem, Southeastern University of Norway, Borre, Norway a zhanghaotian1996@hotmail.com, ${ }^{\text {b } z h a o c h u . y a n g @ c t b u . e d u . c n, ~}{ }^{c}$ tao.dong@usn.no

${ }^{*}$ Corresponding author

Keywords: inclusive design theory, wearable urinalysis device, elderly care Abstract. With the development of the social process, China has entered an age of aging, and the proportion of the disabled elderly is gradually increasing. Therefore, the demand for products of this vulnerable group will gradually increase in the future. This paper proposes the concept and principle of inclusive design theory, and uses this theory to design a wearable urinalysis device. This urinalysis device is designed based on a survey of the physical and psychological demands of the elderly disabled, with consideration of other user groups. This equipment cannot only help elderly disabled to do smart urinalysis, but also help other user groups to monitor their physical condition. It is inclusive and easy to use, can solve the problem of urinalysis for the elderly disabled and full of human care.

\section{Introduction}

China has entered an aging society in 1999. According to a recent report on Shandong's emphasis on aging national education seminar, by 2017, China's population over 60 years-old has reached 240.9 million, accounting for $17.3 \%$ of the total population, and the population over 65 years-old has 158.31 million, accounting for $11.4 \%$. The World Health Organization predicts that by 2050 , China's population over 60 years-old will account for about $35 \%$ of the all, making it one of the most aging countries in the world. With the rapid growth of the elderly population, the number of disabled elderly people is also increasing. As the China Aging Research Center survey shown, by 2015, the number of disabled elderly people in China reached 40 million, accounting for $19 \%$ of the elderly population. Among them, 12 million elderly people are completely disabled seniors who need nursing, accounting for $6.23 \%$ of the elderly population [1].

The disabled elderly is a vulnerable group in society. They may be inconvenient to move, and even stay in bed all the year round, to a certain extent, they need the help of family or nurses. For disabled elderly people, it is difficult for them to control their own physical conditions and to go to the hospital for physical examination. So their demand for physical condition monitoring equipment is very large.

With the aging of the social population, many countries have proposed some similar product design concepts for the demands of the elderly disabled patients, including barrier-free design, transgenerational design, universal design, design for all, and inclusive design [2], etc.. Compared to other design theories, the inclusive design is user-centered, and by reducing the requirements for user capabilities, expanding the applicable objects and applicable environments of the products, improving the tolerance, providing effective and high-quality services to the general public and the elderly, and improving their quality of life. Therefore, the inclusive theory is of great value in the design of products for the elderly disabled [3].

With the rise of smart healthcare, wearable medical devices are playing an increasingly important role in people's daily lives. Wearable medical equipment can be implanted into the human body, bound to the human body, recognize the body's physical characteristics and state, and constantly 
monitor our physical condition, exercise status and metabolic status. The specific functions include real-time monitoring of blood sugar, blood pressure, heart rate, blood oxygen, body temperature, human health indicators such as respiratory rate, but also can play a therapeutic role to a certain extent.

Based on the needs of this age and the concept of inclusive design, we designed a wearable instant urine detection system for the elderly disabled. The system includes wearables (shown in Figure 1) and corresponding inspection software. The system can perform rapid urinalysis on the user, including the detection of five biological indicators of protein, blood, nitrite, leukocytes and glucose to monitor the user's physical condition [4].

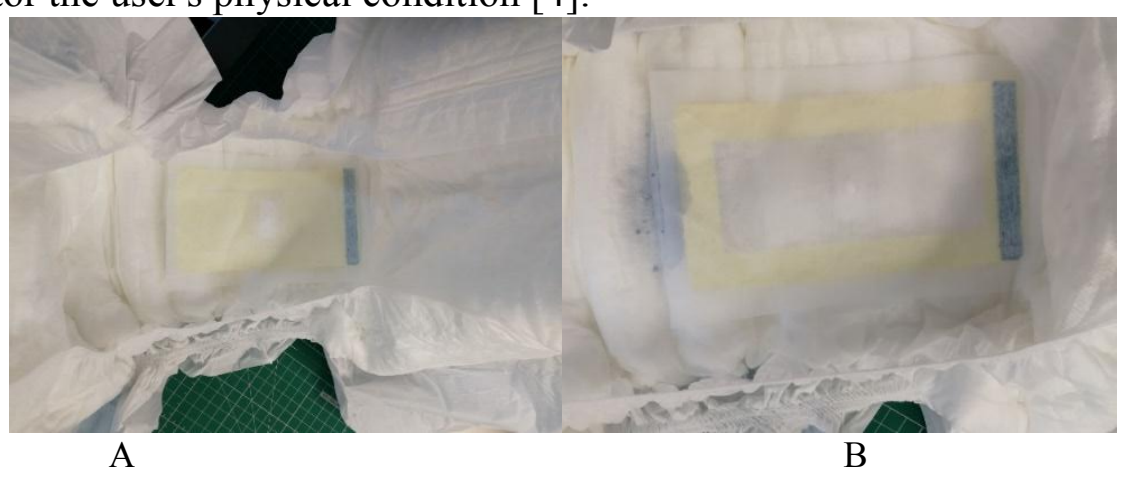

Fig. 1 The wearable device.

(A: Before use; B: After use)

\section{Fundamentals of Inclusive Design Theory}

In 2005, the British Standards Institution defined inclusive design as the design of mainstream products or services can be easily used by as many people as possible without special adaptation or special design [5]. Its application enables designers, manufacturers and service providers to ensure that their products and services meet the widest range of user needs, regardless the limit of age or ability.

The inclusive design can summarized to Double-E principle, easy to use and easy to access. First, the original intention of inclusiveness is to reduce the user's ability requirements. In addition, it needs to improve the user experience and satisfaction of specific people. From a humanistic perspective, inclusive design respects personality and promotes equality for everyone, because inclusive design is designed for existing capabilities rather than defects, and to eliminate differences between different users and care for their psychological feelings.

Many countries have developed inclusive design principles to define or improve inclusiveness in design. And we will introduce the two principles that we insisted in our project.

Firstly, the Paciello Group (TPG) of the United States proposed inclusive design principles in the field of software and interactive interfaces in 2002 [6]:

(1) To ensure that the interface provides an analogous experience for all users, so that people can meet their needs without reducing content.

(2) In any situation, you should ensure that the interface provides a valuable experience.

(3) Follow the conventions and be consistent in application.

(4) Ensure that the user has control. People should be able to access and communicate content in the way they love.

(5) Consider providing people with different ways to accomplish tasks, especially those that are complex or non-standardized.

(6) Help users focus on core tasks, functions, and information by setting priorities in content and layout.

(7) Consider the value of features and how they can improve the experience of different users.

Secondly, we gave an inclusive design principle of wearable device based on our project because there doesn't exist a standard inclusive design principle for wearable device.

(1) Harmless. The equipment cannot harm the users' physical health or mental health. 
(2) Non-invasive. Equipment should not intrude into the human body, to avoid causing discomfort to users.

(3) Easy to use. It is easy to operate and does not require much professional skills.

(4) Respect personal privacy.

(5) Optional. Different people can choose different methods to use the device.

(6) Non-discriminatory. The wearable device should be as trivial as possible, and avoid specialization.

Inclusive design is a comprehensive design approach that meets the needs of most people when it comes to meeting the needs of a certain group of people. In the need for the elderly disabled patients, the use of inclusive product design can not only give them equal social status, but also create more economic benefits for the company as much as possible to achieve a win-win result.

\section{User Characteristics and Demands Analysis}

The concept of wearable urinalysis device is developed regarding to the needs of a nursing home. Many people living in this nursing home are elderly disabled patients who have functional disorder or uracratia and they have to use adult diaper $[7,8]$. The device is designed to monitor their physical condition (the condition of the urinary tract infection) in real time, with consideration of respecting the privacy of patient. Traditional urinalysis are required to go to the hospital, which is time consuming and labor intensive for the elderly. It is more difficult for disabled patients or urinary incontinence patients because they need to use a catheter to insert urine into the reproductive organs. This not only has the potential to cause or exacerbate urinary tract infections, but also affects the dignity of the individual.

For the elderly disabled, their bodies have the following characteristics:

(1) Perceptual function declines, their vision, smell and taste are degrading.

(2) Musculoskeletal function declines, they are slow to respond, slow to move, difficult to bend, and have osteoporosis.

(3) The thinking skills are declining, and their memory and reaction speed are reduced.

And their psychological characteristics are as follows:

(1) Loneliness and helplessness.

(2) Strong self-esteem.

(3) Sensitive and suspicious.

(4) Dependence on others.

(5) Anxious fear, irritability.

(6) Be cautious.

(7) Strongly need to socialize.

Based on their physical and mental characteristics, as well as the requirements of the nursing home, we have the following objectives for the design of wearable urinalysis equipment:

(1) Inclusive.

This product is mainly for wearable urinalysis products designed for the elderly or the disabled. We also hope that it can meet the needs of more people, so we hope that this device can be used for those who have no dysfunction. The crowd provides a portable and simple urinalysis equipment.

(2) Ease of use.

The principle of inclusive design is to reduce the difficulty of use. We will simplify the use steps and give as detailed instructions to improve the experience and satisfaction of elderly disabled patients.

(3) Accuracy.

The product is a portable instant urinalysis device for disease monitoring and pre-diagnosis. We hope that this product's high accuracy will allow users to increase their trust in the product.

(4) Security.

As a medical device, the product should be safe and harmless to the body, and the entire detection system should protect user information and respect privacy, so that can give them a sense of security. 
(5) Cheap.

The ASSURED diagnostic reagent standard issued by the World Health Organization (WHO) proposes that the testing equipment should be cheap, which is also to meet the psychological requirements of elderly patients. They do not want to drag their families because of their own diseases, so the design of this product In the meantime, we will minimize equipment material costs and use costs.

\section{The Wearable Urinalysis Device Based on Inclusive Design Theory}

This device is primarily a wearable instant urinalysis device for elderly disabled urinary tract infection patients. On the hardware, we will provide a urine detection device that can be embedded in the diaper and a color chart. Users can get the results by comparing the color change. We provide an APP on the software, this app can replace visual inspection of urinalysis results and storage of patient test results data, so that doctors and caregivers can observe the patient's physical condition in real time.

\subsection{Hardware design}

The hardware device design of this wearable urinalysis device is shown in Figure 2 and Figure 3. The hardware of this wearable urinalysis device can be embedded in the diaper so that the patient can wear it, and can also be used to provide quick urinalysis for ordinary patients. Not only can users wear it, but they can use the dropper to take urine and then drip into the device for test. The chemical reaction block in the device will chemically react with the urine, and then the reagent block will change color, the patient or caregiver can compare the color displayed by the device with the color chart to read the result. The device is very compact, with a length of $43 \mathrm{~mm}$, a width of 27 $\mathrm{mm}$ and a thickness of $2 \mathrm{~mm}$. As a device embedded in diapers, the device will hardly affect the patient's movements. The main material of the equipment is made of plastic, non-woven fabric, cellophane and other harmless materials, which will not cause harm to the patient's body, and the equipment will not invade the body $[4,9]$.

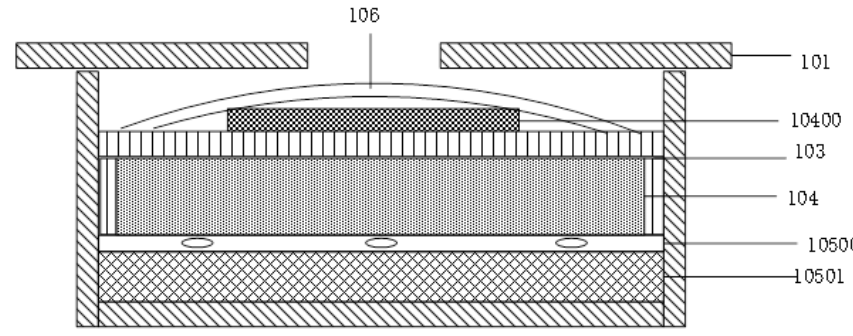

Fig. 2 The overall structure of the wearable device.

(101: Entrance; 103: Urine sampling module;104: Urine self-locking module;10400: Self-locking switch;10500: Isolation layer;10501: Reagent pad module;106: Diversion layer)

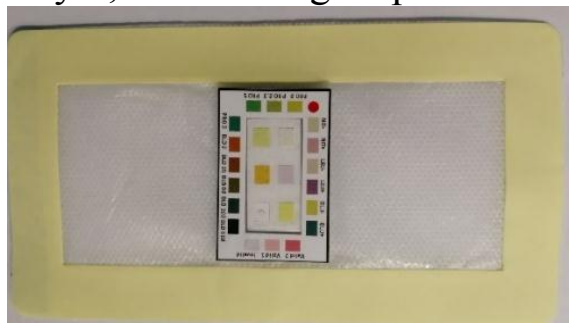

Fig. 3 The device with color chart.

\subsection{Software design}

The design of this software is mainly for the following purposes: first, making the urinalysis results more objective; second, assisting color weak or color blind users to diagnose results; third, storing data in real time, can observe the staged development trend; fourth, help their guardians or doctor monitor the patient's physical condition. The software's interface design adheres to the principle of inclusiveness, it is simple to operate, detailed and easy to use. The specific pages are 
shown in figure 4.

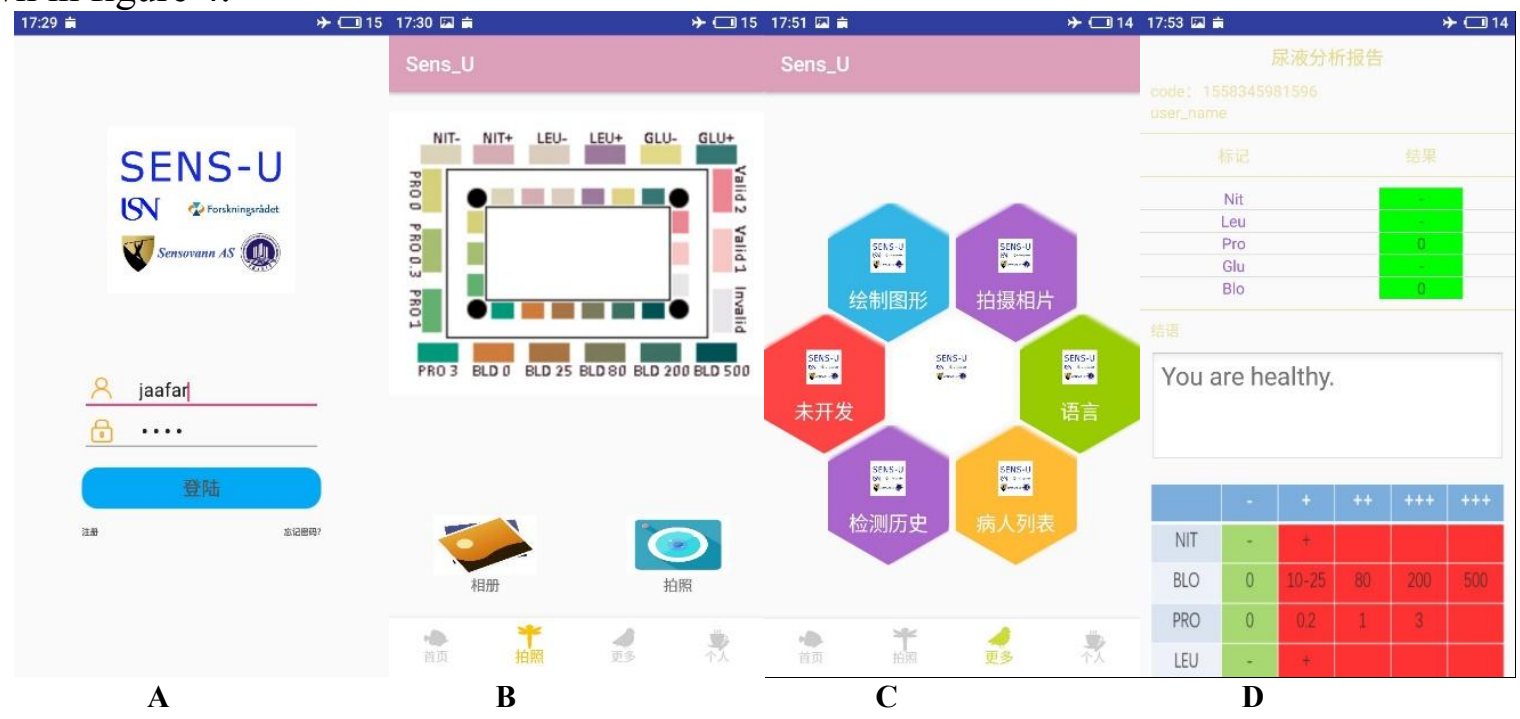

Fig. 4 The main pages of the app.

(A : Home page ; B : Detection Page ; C : Option page ; D : Result Page)

\section{Summary}

In this paper, we introduced the principles and meanings of inclusive design, and designed a wearable urinalysis device for the detection of urinary tract infections by analyzing the physiological and psychological factors of the elderly disabled people. The detection of biological indicators also can be used to monitor other diseases such as diabetes and so on. This urinalysis device consists of two parts, the hardware device and the software. Both parts are designed in accordance with inclusive design principles, not only provide the best service for the elderly disabled, but also it can be used by more people.

\section{Acknowledgment}

This work is supported by the Research Council of Norway projects: FORNY2020: SENS-U:Et nytt og tidsbesparende produkt for prøvetaking og analyse av urin i bleie(No.268481). Also, we would like to thank Huang Jiachang, Deng Shengqiang and Dr. Su Jiafu for the help to improve the paper quality.

\section{References}

[1] Zhao J, Zhou X, Yin Z, et al, Bathing Device for Disabled-the design and Analysis of the Double Lift-Type Bath, international conference on advanced mechatronic systems, 2018.

[2] Ormerod M G , Newton R A , Moving Beyond Accessibility: The Principles of Universal (inclusive) Design as a Dimension in $\mathrm{nD}$ Modelling of the Built Environment, Architectural Engineering \& Design Management, 1.2 (2005)103-110.

[3] Yang J, Su J, Song L, et al, Selection of Manufacturing Enterprise Innovation Design Project Based on Consumer's Green Preferences, Sustainability. 11.5 (2019)1375.

[4] Zhou J, Dong T, Design of a wearable device for real-time screening of urinary tract infection and kidney disease based on smartphone, Analyst. 143.12 (2018) 2812-2818.

[5] Herriott R, When Users Cannot be Included in Inclusive Design, Designing Inclusive Systems. 17 (2012) 165-174.

[6] Information on http://inclusivedesignprinciples.org. 
[7] Karlsen H, Dong T, Suo Z, A Diaper Pad for Diaper-Based Urine Collection and Colorimetric Screening of Urinary Biomarkers, Annals of Biomedical Engineering. 46.36 (2018) 1-9.

[8] Gorina Y, Schappert S , Bercovitz A, et al, Prevalence of Incontinence Among Older Americans, Vital \& health statistics, Series 3, Analytical and epidemiological studies / [U.S. Dept. of Health and Human Services, Public Health Service, National Center for Health Statistics]. 3.36 (2014) 1-33.

[9] Zhang H, Dong T, Identification of Biomarkers with Different Classifiers in Urine Test, international conference of the ieee engineering in medicine and biology society. (2018) 2905-2908. 